\title{
BMJ Open Mathematical modelling in tobacco control research: protocol for a systematic review
}

\author{
Shari Feirman, ${ }^{1,2}$ Elisabeth Donaldson, ${ }^{1,2}$ Jennifer Pearson, ${ }^{1,2}$ \\ Grace Zawistowski, ${ }^{1,3}$ Ray Niaura, ${ }^{1,2,4}$ Allison Glasser, ${ }^{1}$ Andrea C Villanti ${ }^{1,2}$
}

To cite: Feirman S, Donaldson E, Pearson J, et al. Mathematical modelling in tobacco control research: protocol for a systematic review. BMJ Open 2015;5: e007269. doi:10.1136/ bmjopen-2014-007269

- Prepublication history for this paper is available online. To view these files please visit the journal online (http://dx.doi.org/10.1136/ bmjopen-2014-007269).

Received 21 November 2014 Revised 17 March 2015 Accepted 19 March 2015

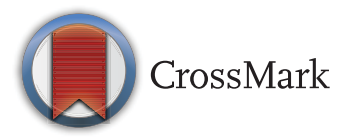

${ }^{1}$ The Schroeder Institute for Tobacco Research and Policy Studies, Legacy, Washington DC, USA

${ }^{2}$ Department of Health, Behavior and Society, Johns Hopkins Bloomberg School of Public Health, Baltimore, Maryland, USA

${ }^{3}$ The George Washington University Milken Institute School of Public Health ${ }^{4}$ Lombardi Comprehensive Cancer Center, Georgetown University Medical Center, Washington DC, USA

Correspondence to Andrea C Villanti; avillanti@legacyforhealth.org

\section{ABSTRACT}

Introduction: Tobacco control researchers have recently become more interested in systems science methods and mathematical modelling techniques as a means to understand how complex inter-relationships among various factors translate into population-level summaries of tobacco use prevalence and its associated medical and social costs. However, there is currently no resource that provides an overview of how mathematical modelling has been used in tobacco control research. This review will provide a summary of studies that employ modelling techniques to predict tobacco-related outcomes. It will also propose a conceptual framework for grouping existing modelling studies by their objectives.

Methods and analysis: We will conduct a systematic review that is informed by Cochrane procedures, as well as guidelines developed for reviews that are specifically intended to inform policy and programme decision-making. We will search 5 electronic databases to identify studies that use a mathematical model to project a tobacco-related outcome. An online data extraction form will be developed based on the ISPORSMDM Modeling Good Research Practices. We will perform a qualitative synthesis of included studies.

Ethics and dissemination: Ethical approval is not required for this study. An initial paper, published in a peer-reviewed journal, will provide an overview of our findings. Subsequent papers will provide greater detail on results within each study objective category and an assessment of the risk of bias of these grouped studies.

\section{INTRODUCTION}

In its early years, the field of tobacco control benefited from studies examining the aetiology of tobacco-related disease. Today, the research paradigm in the field relies on a broader perspective that takes into account the complete system within which tobacco use and tobacco control interventions operate. $^{1}{ }^{2}$ Acknowledging the complex drivers of tobacco use and related diseases, tobacco control researchers have become

\section{Strengths and limitations of this study}

- To the best of our knowledge, this is the first review to systematically synthesise studies that have employed mathematical models to predict tobacco-related outcomes.

- This study will produce a conceptual framework for grouping existing modelling studies, which will serve as the foundation for future analyses of these data.

- The results of this study may inform future mathematical models in the field of tobacco control.

- This review will be limited to English language and peer-reviewed articles.

increasingly interested in systems science methods. ${ }^{3} 4$

One approach used in systems science research is mathematical modelling which, broadly conceived, illustrates and attempts to quantify how a system's components are inter-related and how outcomes of interest might be affected when a system undergoes change. ${ }^{3}$ In tobacco control research, such models have been used to understand the future scope of disease burden, ${ }^{5-7}$ the potential effects of policies and regulations, ${ }^{8-10}$ and the economic consequences of interventions. ${ }^{11-13}$ However, there is currently no resource that provides an overview or synthesis of how these modelling techniques have been developed and used in tobacco control. This gap limits the ability of researchers to learn from and improve on existing models. Moreover, modelling approaches have been heterogeneous in their methods and even though they attempt to predict similar types of outcomes (eg, lung cancer, qualityadjusted life years), results have not been adequately synthesised, weakening their ability to contribute to the evidence base for regulatory policy decisions. This review will address this gap by providing a summary of 
modelling techniques that have been used in tobacco control research to predict tobacco-related outcomes. It will also propose a conceptual framework for grouping existing modelling studies by their objectives, which will serve as the foundation for future analyses of these data.

This effort is intended to inform regulatory decisionmaking. The Center for Tobacco Products at the US Food and Drug Administration has expressed an interest in modelling the impact of its policies. ${ }^{14}$ The results of this review will help decision-makers at the national, state and local levels to gain a better understanding of the scope, content and quality of existing modelling studies.

\section{METHODS AND ANALYSIS}

The Cochrane Collaboration provides rigorous guidelines for conducting systematic reviews. ${ }^{15}$ However, these guidelines were developed to inform systematic reviews that investigate the effectiveness of healthcare interventions. ${ }^{16}$ Thus, while the Cochrane approach to conducting systematic reviews will inform the methodology of this review, ${ }^{15}$ we will also draw on guidelines from reviews that are specifically intended to inform policy and programme decision-making. ${ }^{17}$ This protocol reports on recommended items to address in a systematic review protocol, in compliance with the preferred reporting items for systematic reviews and meta-analyses protocols (PRISMA-P). ${ }^{18}$

\section{Eligibility criteria}

Eligible studies must use a mathematical model to project a tobacco-related outcome. 'Projection' is defined as the use of data-real or hypothetical-to estimate an outcome prospectively beyond the date from when the data were collected. Mathematical models with differing structures, purposes and outcomes will be eligible for this review, including system dynamics models, ${ }^{19}$ dynamic simulations, ${ }^{20}$ state-transition models, ${ }^{21}$ decision trees, ${ }^{22}$ microsimulations ${ }^{23}$ and economic models. ${ }^{24}$

Outcomes must estimate the prevalence of tobacco use (including changes in initiation or cessation), tobaccorelated health outcomes, and/or tobacco-related costs. Tobacco-related health outcomes are broadly defined to include any outcome that has evidence of a relationship with tobacco use, including outcomes for which the Surgeon General finds inadequate evidence to conclude that a causal relationship is present. ${ }^{25}{ }^{26}$ Eligible models may include any analytic time horizon. Models with lifetime horizons will be included, even if the authors do not report outcomes to a specific date. Studies assessing nontobacco related outcomes, such as alcohol use or obesity prevalence, will be included if a tobacco-related outcome is also examined. Real and hypothetical populations contributing data for these modelling studies may be of any age, gender, race/ethnicity, and from any geographic location. Only peer-reviewed, published literature will be eligible for inclusion.
Models that project only retrospectively, such as those that analyse the historical burden of disease, will be excluded from this review, as will studies that model individual smoker trajectories that do not also project population-level outcomes. Additionally, animal studies, human genetics studies, and posters and abstracts without full-text records will be excluded from this review. This review will be limited to English language studies.

\section{Search methods for identification of studies}

A comprehensive search strategy, developed in consultation with a university librarian and group of tobacco control experts, will be used to identify eligible papers (see table 1). We developed the search strategy by identifying medical subject headings for recent tobacco simulation model publications. ${ }^{8}{ }^{27-29}$ A search strategy was developed to address the unique features and indexing of each of the five electronic databases: PubMed, Embase, EconLit, CINAHL and PsychInfo. These databases were selected based on recommendations from the university librarian, and these will be searched with no date restrictions. We will use EndNote X6 to manage records throughout this review.

\section{Data collection and analysis}

\section{Selection of studies}

Eligibility criteria will be piloted by multiple reviewers prior to the title and abstract review, and refined to ensure consistency across reviewers. In the first stage of study selection, coders will independently review the title and abstract of each included record. In order to be selected for the full-text review, titles and abstracts must refer to any type of tobacco product or tobacco use, and indicate that the study projected a tobacco-related outcome, as defined above. After the double review, each record will be categorised using the following criteria: (1) both authors agree on inclusion; (2) one author recommends inclusion; (3) both authors are unsure; (4) one author recommends exclusion and the other is unsure or (5) both authors agree on exclusion. Full-text articles for abstracts classified as 1 or 2 will be retrieved. Records classified as 3 or 4 will be discussed further by two reviewers and if needed, a third reviewer will be consulted to determine whether the record should be included in the full-text review. Records classified as 5 will not be considered for full-text review.

Using the same pilot testing, double review and categorisation process, two coders will review the full texts of selected articles. After independent review of the articles, the coders will meet to categorise each article using the criteria listed above; again, records classified as 1 or 2 will be considered as included studies. At this time, the coders will resolve discrepancies and record the reasons for record exclusions in a log. If an article is excluded for multiple reasons, only the primary reason for exclusion will be noted. The hierarchy for identifying 


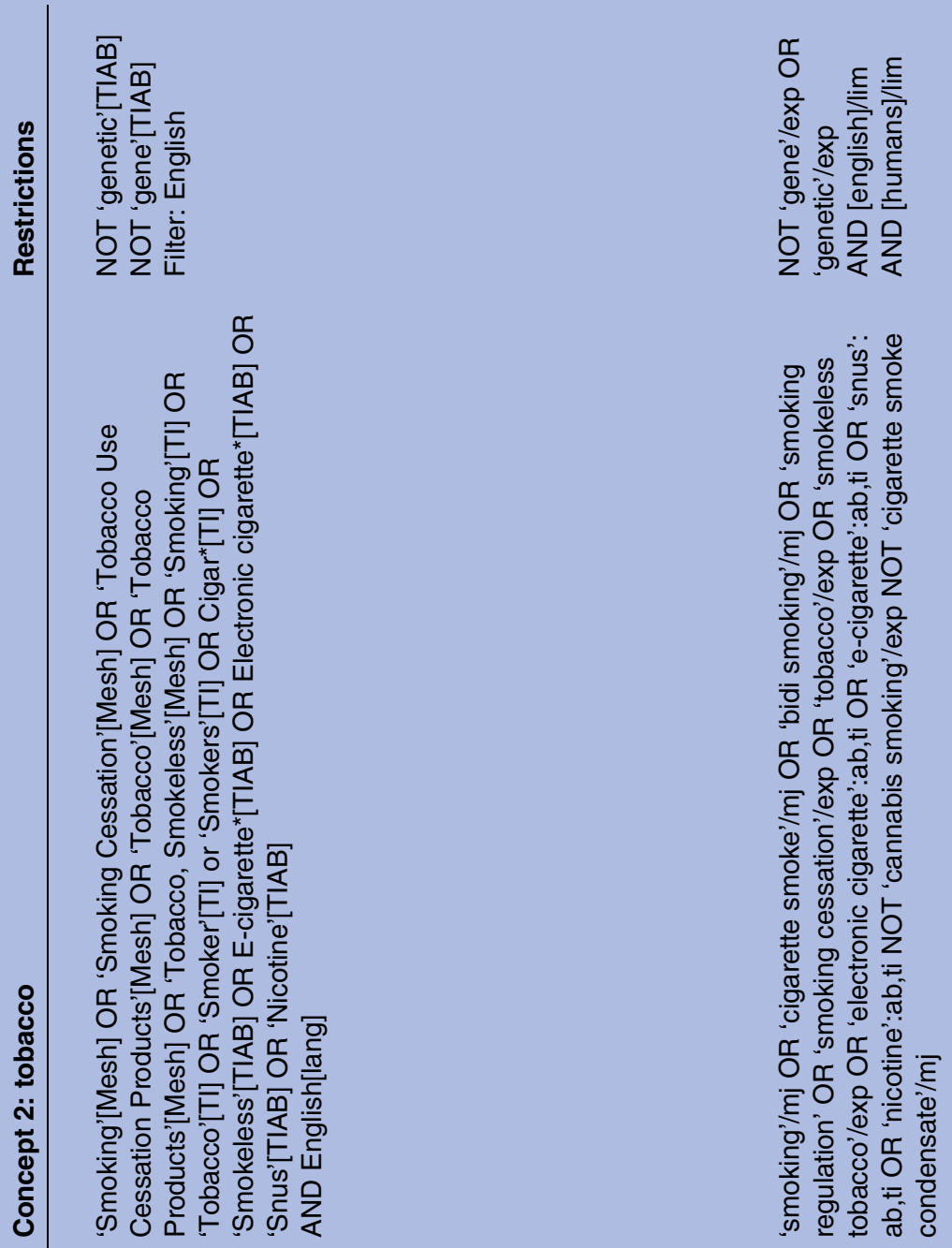

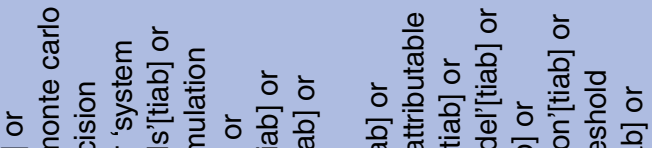

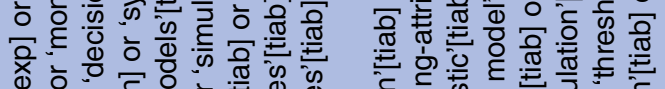

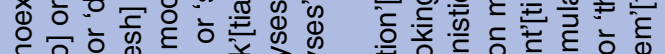

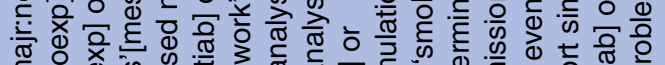

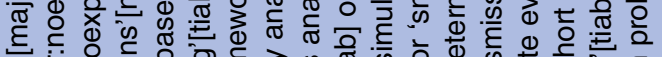

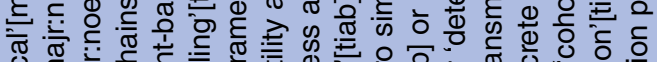

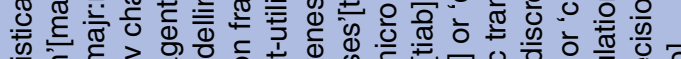
क्ञ

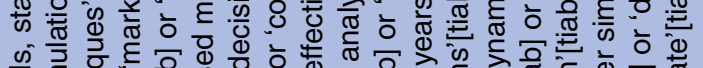
क) ठ

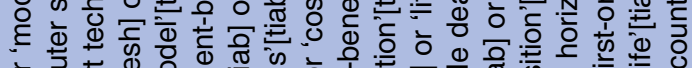

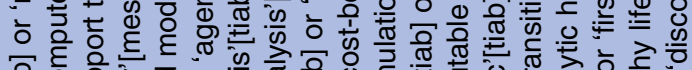

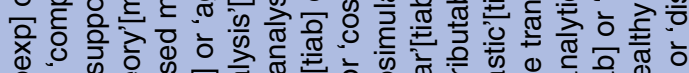
o

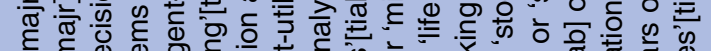

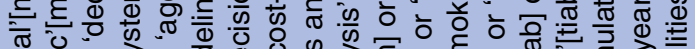
ब。 㐫 ब $\underset{\mathbb{\pi}}{\mathbb{2}}$

๘े

등 응

몽

峞

윯

은 옹 $\frac{0}{0}$

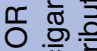

인

항 응

की in है

品 $\frac{\infty}{0}$

잉 잉

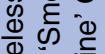

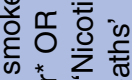

$\times$ 范

讨 0

잉

8 is के

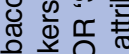

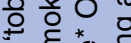

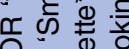

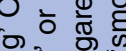

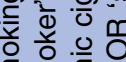

है

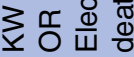

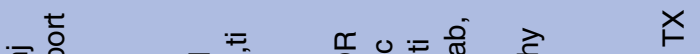

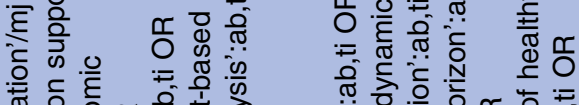

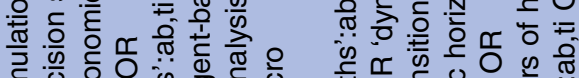

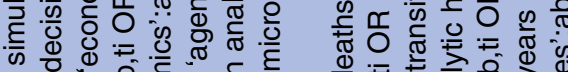

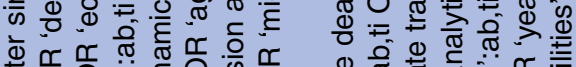

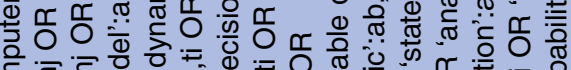
है

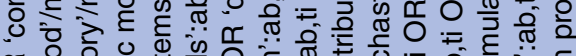
穴

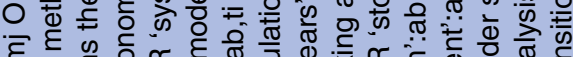

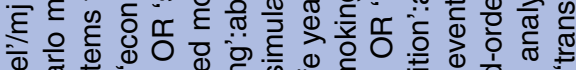
ब。

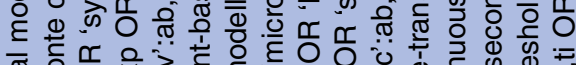

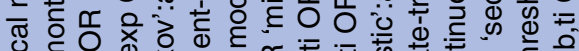

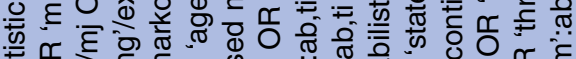

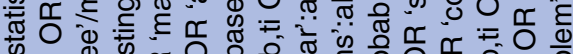
के 范

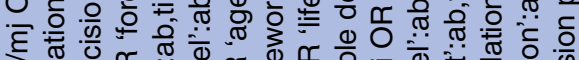

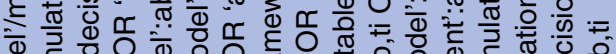

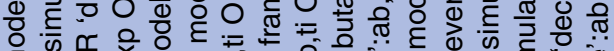

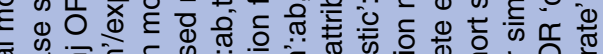
뜽 뜽 홍

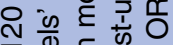

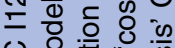
O

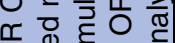
○

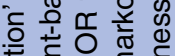
का

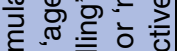
के

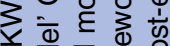

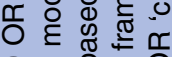

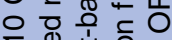
5 क O 뜽 을

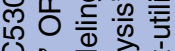
0 is $\frac{0}{2} \frac{1}{\mathrm{~T}}$

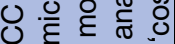

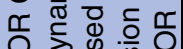
क

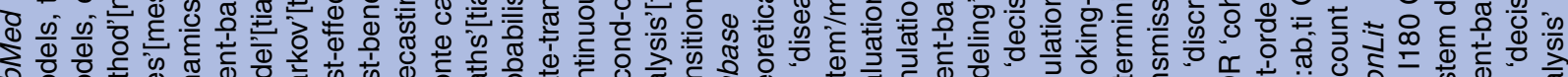

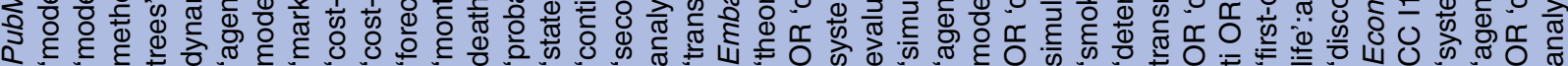

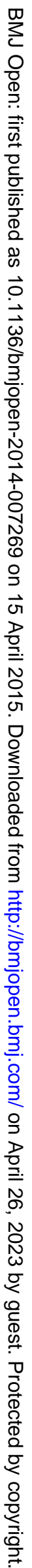




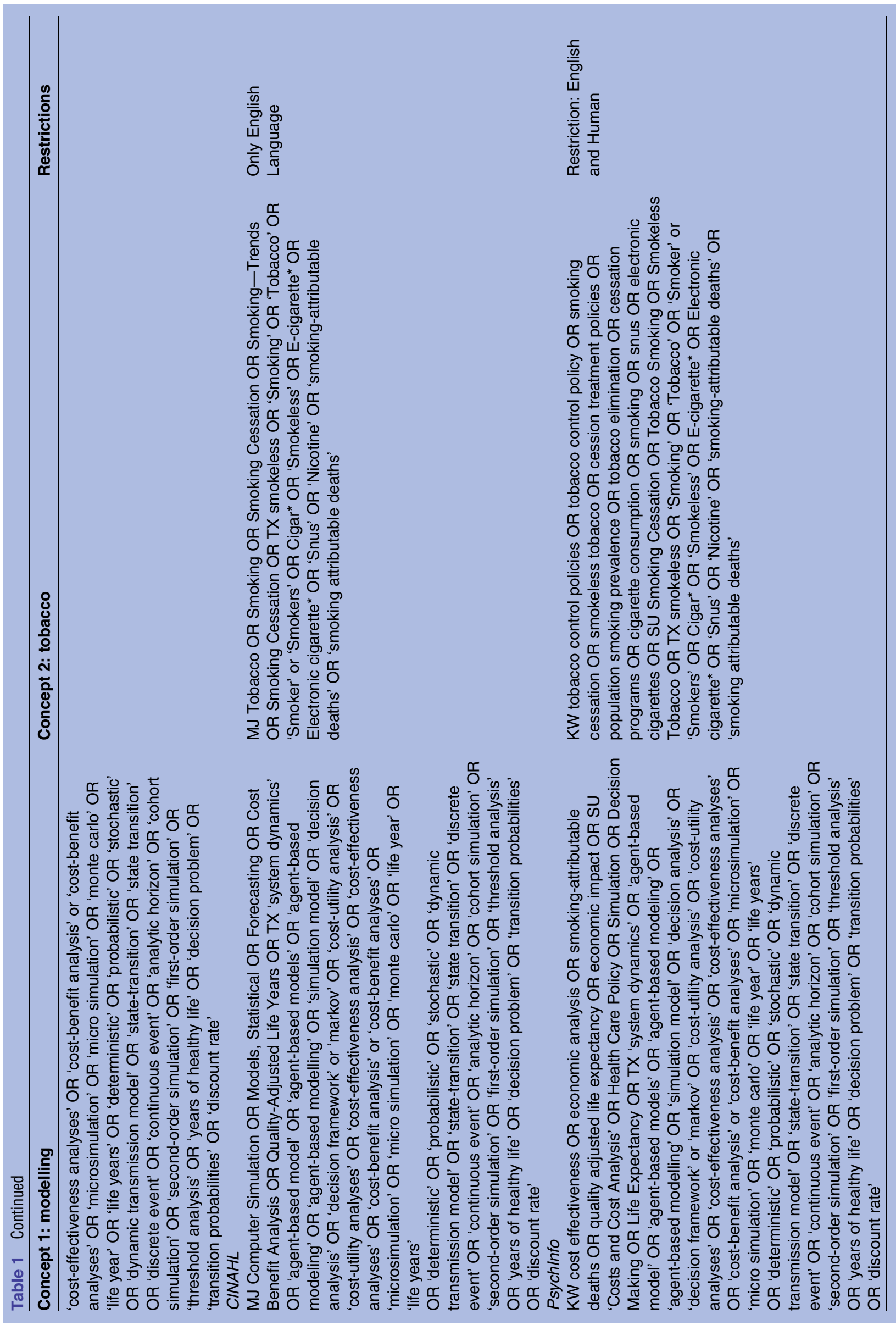


the reason for exclusion when multiple reasons exist will be as follows: (1) the article was not available in English; (2) the article did not contain an original analysis; (3) the study did not include a projected outcome; or (4) the study did not include a tobacco-related outcome. The remaining studies will be retained for inclusion in the systematic review.

\section{Data extraction}

The ISPOR-SMDM Modeling Good Research Practices Task Force published a series of papers that provide recommendations for best practices in developing and reporting on mathematical models. ${ }^{30-36}$ An online data extraction form will be developed based on the ISPOR-SMDM Modeling Good Research Practices ${ }^{31}$ and will contain items regarding the study objective, purpose, model structure, inputs and outcomes. We will approach form development with the expectation that model objectives will fall into one or more of three categories: (1) to evaluate the effects of a population-level policy, legislative, or regulatory action ('policy study'); (2) to assess the economic implications of a policy or programme ('economic study'); and (3) to estimate the effect that tobacco use changes or trends in tobacco-related disease would have on future morbidity or mortality outcomes ('disease study'). Owing to the variability in the types of models and inputs assessed in this review, and the novel nature of this type of review, this form will undergo significant pilot testing. During this process, the form will be revised to facilitate data collection for technical and conceptual aspects of included papers.

\section{Data synthesis}

After the data are collected, we will perform a qualitative synthesis of the included studies. An initial paper will provide an overview of our findings. This overview will include a conceptual framework, based on the model objective categories we identified, that will be used to organise existing tobacco modelling studies. We will also identify methodological commonalities across papers. Subsequent papers will provide greater detail on results within each study objective category and an assessment of the risk of bias of these grouped studies; to conduct these analyses, we will perform additional rounds of data extraction with subgroups of the included studies. Given the large scope of this review and heterogeneity of included model structures and outcomes, we do not expect to conduct meta-analyses of model results. An additional outcome of this effort will be to determine if meta-analyses might be possible at some future point by identifying homogenous subgroups of studies, and recommending common methods and measures for ongoing and future studies that would permit meta-analyses and data pooling.

\section{POTENTIAL LIMITATIONS}

Potential limitations of this study include the risk of bias introduced by restricting the search to English language studies and peer-reviewed literature. Our ability to conduct quality assessments of the included studies may also ultimately be limited due to the nature of mathematical modelling methods. That is, there are numerous ways that a modeller can choose to structure his or her model, and this decision impacts the model outcomes. As noted in a report of the ISPOR-SMDM Modeling Good Research Practices Task Force, many a time choices regarding model structure "are made on the basis of expert opinion, or influenced by concerns for simplicity, feasibility of implementation, and so on. This process leaves much room for uncertainty, but it is very difficult to quantify and analyse this uncertainty." ${ }^{31}$ While this characteristic of mathematical modelling may make it difficult to assess the internal validity of some models, the results of this systematic review will allow us to assess the cross validity of models by comparing different types of models that address similar research questions. ${ }^{32}$

Acknowledgements The authors would like to thank Donna Hesson at the Welch Medical Library for her assistance with the search strategy and colleagues at the Schroeder Institute for their feedback on earlier versions of the protocol.

Contributors ACV conceived the idea for this study. SF and ED drafted the protocol and developed the search strategy, with support from ACV, JP, GZ and RN. ACV, JP, GZ and ED will conduct the title and abstract review. SF, ED and $A G$ will perform data extraction. ACV is the guarantor of the review. All authors provided input on inclusion and exclusion criteria.

Funding This work was supported by the Schroeder Institute for Tobacco Research and Policy Studies, Legacy. All authors were employed by Legacy.

Competing interests None declared.

Provenance and peer review Not commissioned; externally peer reviewed.

Data sharing statement This study is a systematic review of the literature and thus, uses data that are already publicly available.

Ethics and dissemination This review synthesises published literature; ethical approval is not required for this study. The results of this study will be disseminated in a peer-reviewed journal.

Open Access This is an Open Access article distributed in accordance with the Creative Commons Attribution Non Commercial (CC BY-NC 4.0) license, which permits others to distribute, remix, adapt, build upon this work noncommercially, and license their derivative works on different terms, provided the original work is properly cited and the use is non-commercial. See: http:// creativecommons.org/licenses/by-nc/4.0/

\section{REFERENCES}

1. Abrams DB. Transdisciplinary paradigms for tobacco prevention research. Nicotine Tob Res 1999;1(Suppl 1):S15-23.

2. Abrams DB, Leslie F, Mermelstein R, et al. Transdisciplinary tobacco use research. Nicotine Tob Res 2003;5(Suppl 1):S5-10.

3. Mabry PL, Marcus SE, Clark PI, et al. Systems science: a revolution in public health policy research. Am J Public Health 2010;100:1161-3.

4. Luke DA, Stamatakis KA. Systems science methods in public health: dynamics, networks, and agents. Annu Rev Public Health 2012;33:357-76.

5. Murray CJ, Lopez AD. Global mortality, disability, and the contribution of risk factors: Global Burden of Disease Study. Lancet 1997;349:1436-42.

6. Brown CC, Kessler LG. Projections of lung cancer mortality in the United States: 1985-2025. J Natl Cancer Inst 1988;80:43-51.

7. Brennan $\mathrm{P}$, Bray I. Recent trends and future directions for lung cancer mortality in Europe. Br J Cancer 2002;87:43-8.

8. Levy DT, Pearson JL, Villanti AC, et al. Modeling the future effects of a menthol ban on smoking prevalence and smoking-attributable deaths in the United States. Am J Public Health 2011;101:1236-40. 
9. Guerrero F, Santonja FJ, Villanueva RJ. Analysing the Spanish smoke-free legislation of 2006: a new method to quantify its impact using a dynamic model. Int J Drug Policy 2011;22:247-51.

10. Tengs TO, Ahmad S, Moore R, et al. Federal policy mandating safer cigarettes: a hypothetical simulation of the anticipated population health gains or losses. J Policy Anal Manage 2004;23:857-72.

11. Secker-Walker RH, Holland RR, Lloyd CM, et al. Cost effectiveness of a community based research project to help women quit smoking Tob Control 2005;14:37-42.

12. Cummings SR, Rubin SM, Oster G. The cost-effectiveness of counseling smokers to quit. JAMA 1989;261:75-9.

13. Lal A, Mihalopoulos $C$, Wallace $A$, et al. The cost-effectiveness of call-back counselling for smoking cessation. Tob Control 2013;23:437-42.

14. Polosa $\mathrm{R}$, Rodu $\mathrm{B}$, Caponnetto $\mathrm{P}$, et al. A fresh look at tobacco harm reduction: the case for the electronic cigarette. Harm Reduct $J$ 2013;10:19.

15. Higgins J, Green S. Cochrane handbook for systematic reviews of interventions version 5.1.0 [updated March 2011]. The Cochrane Collaboration, 2011.

16. Cochrane Handbook For Systematic Reviews of Interventions. http:// community.cochrane.org/handbook (accessed 10 Mar 2015).

17. Greenhalgh T, Wong G, Westhorp G, et al. Protocol-realist and meta-narrative evidence synthesis: evolving standards (RAMESES) BMC Med Res Methodol 2011;11:115.

18. Shamseer L, Moher D, Clarke M, et al. Preferred reporting items for systematic review and meta-analysis protocols (PRISMA-P) 2015: elaboration and explanation. BMJ 2015;349:g7647.

19. Tobias MI, Cavana RY, Bloomfield A. Application of a system dynamics model to inform investment in smoking cessation services in New Zealand. Am J Public Health 2010;100:1274-81.

20. Warner KE, Méndez D. Accuracy and importance of projections from a dynamic simulation model of smoking prevalence in the United States. Am J Public Health 2012;102:2045-8.

21. Tosteson AN, Weinstein MC, Williams LW, et al. Long-term impact of smoking cessation on the incidence of coronary heart disease. $A m \mathrm{~J}$ Public Health 1990;80:1481-6. http://www.ncbi.nlm.nih.gov/pubmed/ 2240335 (accessed 24 Feb 2015).

22. Boyd KA, Briggs AH. Cost-effectiveness of pharmacy and group behavioural support smoking cessation services in Glasgow. Addiction 2009;104:317-25.

23. Jeon J, Meza R, Krapcho M, et al. Chapter 5: actual and counterfactual smoking prevalence rates in the U.S. population via microsimulation. Risk Anal 2012;32(Suppl 1):S51-68.

24. Halpern MT, Khan ZM, Young TL, et al. Economic model of sustained-release bupropion hydrochloride in health plan and work site smoking-cessation programs. Am J Health Syst Pharm 2000;57:1421-9. http://www.ncbi.nlm.nih.gov/pubmed/10938982 (accessed 24 Feb 2015).

25. The Health Consequences of Involuntary Exposure to Tobacco Smoke: a Report of the Surgeon General-Executive Summary. U.S. Department of Health and Human Services, Centers for Disease Control and Prevention, Coordinating Center for Health Promotion, National Center for Chronic Disease Prevention and Health Promotion, Office on Smoking and Health, 2006.

26. The Health Consequences of Smoking: a Report of the Surgeon General. Washington, DC: Dept. of Health and Human Services, Centers for Disease Control and Prevention, National Center for Chronic Disease Prevention and Health Promotion, Office on Smoking and Health, 2004.

27. Levy DT, Boyle RG, Abrams DB. The role of public policies in reducing smoking: the Minnesota SimSmoke tobacco policy model. Am J Prev Med 2012;43(5 Suppl 3):S179-86.

28. Mendez D, Warner KE, Courant PN. Has smoking cessation ceased? Expected trends in the prevalence of smoking in the United States. Am J Epidemiol 1998;148:249-58.

29. Levy D, Gallus S, Blackman K, et al. Italy SimSmoke: the effect of tobacco control policies on smoking prevalence and smoking attributable deaths in Italy. BMC Public Health 2012;12:709.

30. Briggs $\mathrm{AH}$, Weinstein MC, Fenwick EA, et al. Model parameter estimation and uncertainty: a report of the ISPOR-SMDM Modeling Good Research Practices Task Force-6. Value Health 2012;15: 835-42.

31. Caro JJ, Briggs AH, Siebert $\mathrm{U}$, et al. Modeling good research practices-overview: a report of the ISPOR-SMDM Modeling Good Research Practices Task Force-1. Med Decis Making 2012;32:667-77.

32. Eddy DM, Hollingworth W, Caro JJ, et al. Model transparency and validation: a report of the ISPOR-SMDM Modeling Good Research Practices Task Force-7. Med Decis Making 2012;32:733-43.

33. Karnon J, Stahl J, Brennan A, et al. Modeling using discrete event simulation: a report of the ISPOR-SMDM Modeling Good Research Practices Task Force-4. Value Health 2012;15:821-7.

34. Pitman R, Fisman D, Zaric GS, et al. Dynamic transmission modeling: a report of the ISPOR-SMDM Modeling Good Research Practices Task Force-5. Value Health 2012;15:828-34.

35. Roberts M, Russell LB, Paltiel AD, et al. Conceptualizing a model: a report of the ISPOR-SMDM Modeling Good Research Practices Task Force-2. Value Health 2012;15:804-11.

36. Siebert $\mathrm{U}$, Alagoz O, Bayoumi AM, et al. State-transition modeling: a report of the ISPOR-SMDM Modeling Good Research Practices Task Force-3. Value Health 2012;15:812-20. 